\title{
Controller design techniques applied for a case of a linear fractional system
}

\author{
Rim Jaouani, Kaouther Laabidi \\ System Analyzis and Command Laboratory ACS \\ National Engineering School of Tunis \\ Tunis, Tunisia \\ jaouanirima@gmail.com, kaouther.laabidi@enit.rnu.tn
}

\begin{abstract}
- the purpose of this paper is to present tow methods to synthesize fractional order control for a given type of Fractional Order System FOS model. Two controllers are proposed for this class of FOS: Fractional Order Proportional Integrator (FO-PI) controller and Proportional Integrator PI. A practical and systematic tuning procedure has been developed for these controllers. In fact the paper presents an extension of some results developed in the simulation of integer order plants to FOS using the state representation; Computer simulation of the circuit model by Matlab was used to prove the relevance of our work and to show the effectiveness of the proposed controller design strategy and the robustness of the FO-PI controller compared to the classic PI under the same number of design parameters and the same specifications.
\end{abstract}

Keywords-; fractional calculus, controller design, fractional order PI controller, integer order PI controller, fractional system.

\section{INTRODUCTION}

Due to the better understanding of fractional calculus [1], fractional order differential equations seems be more adequate to describe real world systems than those of integer order models [2, 3].

Fractional systems are defined in terms of their spectra shape which is proportional to reciprocal frequency and which is generally called $1 / \mathrm{F}$ beta spectra $[4,5]$.

Thus the concept of differentiation to an arbitrary order was defined in the 19th century by using not only integer but also non integer order. Fractional calculus can be considered to be a generalization of integration and differentiation of the integer order expressions to the non-integer order one. The most frequently used integro-differential definitions are Grünwald-Letnikov, Riemann-Liouville and Caputo expressions which were given in [6].

In recent years considerable attention has been given to the fractional calculus used in modeling and control of various kinds of physical systems. As a result, many real systems have been modeled by fractional order systems [6]. the dynamic model which governs the phenomenon of transposition of "fractal robustness" is a fractional linear equation [7], In fact some important studies dealing with the applications of the fractional calculus to the control system have been done in $[8,9]$.

Our contribution concerns the control of fractional order systems. The control requires the preliminary theoretical knowledge of the fractional calculation mention in the first section which will be useful not only for the control phase but also for the analysis and the simulation of these type of systems, In section two we focus on the analyze and representation of a fractional system, two regulators will be synthesized in section three with the aim of controlling the system discussed.

\section{ANALYZE OF THE FRACTIONAL ORDER SYSTEM}

\section{A. Frequency analyze}

Many of real physical system can be better represented by an explicit fractional transfer function. The FOS discussed in this paper has the following form :

$$
P(s)=\frac{1}{s\left(T s^{\alpha}+1\right)}
$$

This generalized fractional capacitor membrane model does play an important role in describing the dielectric behavior of membranes cells; tissues and a variety of biological material for example, nerve muscle [10].

\section{B. Phase and Gain of the system discussed}

Writing the transfer function in the frequency domain, we focus on the following shape

$$
p(j w)=\frac{1}{j w\left(\left(T(j w)^{\alpha}+1\right)\right.}
$$

The fractional puissance of $j w$ can be written as given in $[11]$ :

$$
(j w)^{\alpha}=w^{\alpha} j^{\alpha}=w^{\alpha}\left[e^{j\left(\frac{\pi}{2}+2 n \pi\right)}\right]^{\alpha}=w^{\alpha}\left[e^{j\left(\frac{\pi}{2} \alpha+2 n o \pi\right)}\right]
$$

After developing, both the gain and the module have the following form: 


$$
\left\{\begin{array}{l}
\mid P(j w)=\sqrt{\left(T w \cos \frac{\alpha \pi}{2}+w\right)^{2}+\left(T w \sin \frac{\alpha \pi}{2}\right)^{2}} \\
\operatorname{Arg}\left(P(j w)=-\tan ^{-1}\left(\frac{T w_{c}{ }^{\alpha} \sin \left(\frac{\alpha \pi}{2}\right)}{T w_{c}{ }^{\alpha}\left(\cos \left(\frac{\alpha \pi}{2}\right)+1\right.}\right)-\frac{\pi}{2}\right.
\end{array}\right.
$$

As an illustration example, we have chosen a fractional system which has the following parameters: $T=0.4, \alpha=1.4, w_{c}=10^{\circ}$.

The Figure 1 shows the bode plot of the system represented by the equation system (4).
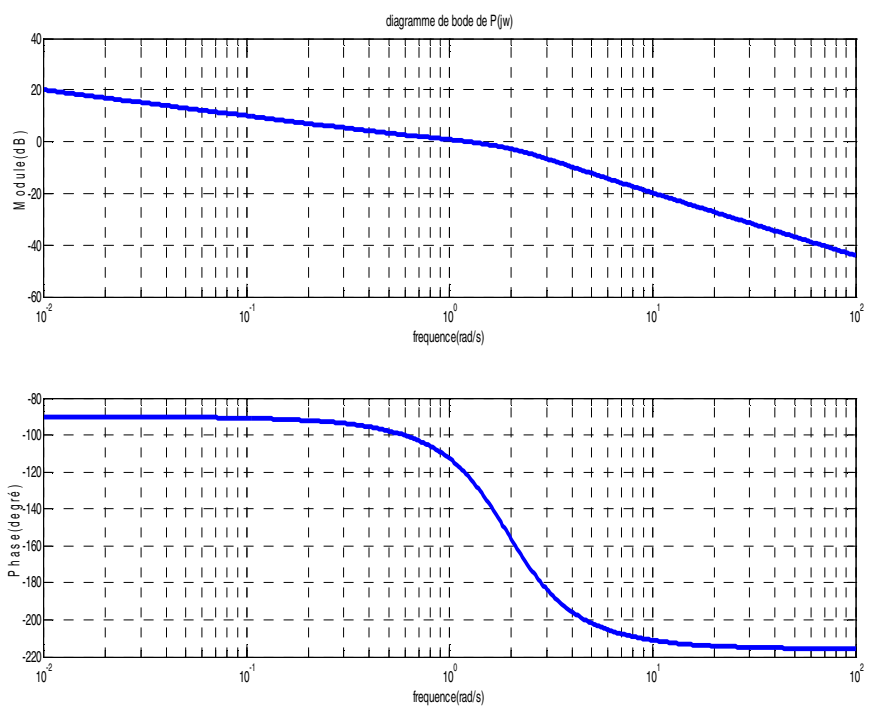

Fig1: bode diagram of the FOS

As seen from the phase plot of the FOS, the phase down to $-180 \mathrm{deg}$ for higher frequencies. The phase margin $\phi_{m}=56^{\circ}$ is achieved at the frequency of $w_{c}=1 \mathrm{rd} / \mathrm{s}$.

\section{CONTROL SPECIFICATIONS AND TUNNING}

The idea of this procedure is to propose an exercise book predefines for the opened loop from which we are going to try to determine the expression of the specified regulator. This exercise book is only all these conditions to be put on the phase and the module of the wished system, these last conditions are translated to a system of equation, the resolution of this system allows to insure the performances wished provided that the number of the parameters of the regulator is lower or equals among equation. In our case we are going to limit itself to regulators having in most three parameter, for the evaluation of these parameters, we are going to put then three conditions to have the behavior, these three conditions are according to the terms of the controller [10].

The first condition deal with the specification of the margin of phase at the corner frequency, in fact the phase of the regulated system $G\left(j w_{c}\right)$ does not have to exceed $180^{\circ}$.
For a system $P(s)$ in waterfall with a regulator $C(s)$, the open loop is:

$$
G(s)=C(s) P(s)
$$

Thus, the argument of $G(j w)$ has to verify the following equation:

$$
\begin{aligned}
\operatorname{Arg}[G(j w)]= & \operatorname{Arg}\left[C\left(j w_{c}\right) P\left(j w_{c}\right)\right] \\
& =-\pi+\phi_{m}
\end{aligned}
$$

Where $w_{c}$ the corner frequency wished and $\phi_{m}$ is the margin of required phase.

The second condition has to guarantee the constancy of the phase for the opened loop for the $w \geq w_{c}$, what allows a better robustness to the change of the gain and the overtaking This condition is translated by the equation (7).

$$
\left(\frac{d \operatorname{Arg}\left[C\left(j w_{c}\right) P\left(j w_{c}\right)\right]}{d w_{c}}\right)_{w=w_{c}}=0
$$

The third condition influences on the amplitude of the open loop; the equation (8) insures the passage by zero of the module at the wished corner frequency.

$$
\left|G\left(j w_{c}\right)_{d B}=\right| C\left(j w_{c}\right) P\left(j w_{c}\right)||=0
$$

\section{A. Fractional Order [PI] controller design}

We always considering the fractional system described by the equation (1), the regulator FO-[ PI] is of the following shape:

$$
C_{1}(s)=k_{p}\left(1+\frac{k_{i 1}}{s}\right)^{\lambda}
$$

The phase and the gain of the proposed FO-[PI] controller can be given as follows:

$$
\left\{\begin{array}{l}
\left|C_{1}(j w)\right|=k_{p 1}\left(1+\left(\frac{-k_{i 1}}{w_{c}}\right)^{2}\right)^{\frac{\lambda}{2}} \\
\operatorname{Arg}\left(C_{1}(j w)\right)=\lambda \operatorname{arctg}\left(\frac{-k_{i 1}}{w_{c}}\right)^{2}
\end{array}\right.
$$

Let us look now for the parameters of the regulator (9) from the three specifications previously mentioned.

The first condition (6) allows writing: 


$$
\begin{gathered}
\operatorname{Arg}\left(G_{1}(j w)=-\tan ^{-1}\left(\frac{T w_{c}^{\alpha} \sin \frac{\alpha \pi}{2}}{1+T w_{c}^{\alpha} \cos \frac{\alpha \pi}{2}}\right)-\frac{\pi}{2}+\lambda \operatorname{arctg}\left(\frac{-k_{i 1}}{w_{c}}\right)^{2}\right. \\
=-\pi+\phi_{m}
\end{gathered}
$$

We can then establish from the expression (11) a relation between $k_{i 1}$ and $\lambda$.

$k_{i 1}=-w_{c} \tan \left(\frac{1}{\mu}\left(\phi_{m}-\frac{\pi}{2}+\tan ^{-1}\left(\frac{T w_{c}^{\alpha} \sin \frac{\alpha \pi}{2}}{1+T w_{c}^{\alpha} \cos \frac{\alpha \pi}{2}}\right)\right)\right)$

From the first condition we pulled the equation ( 12 ), now we need a second equation having the same unknowns as that one to found these parameters, thus exploiting the equation ( 7 ) this equation asks for the calculation of the derivative of the tangent function .

Thus, from the second condition we can write

$$
\left(\frac{d \operatorname{Arg}[C(j w)]}{d w}\right)_{w=w_{c}}=\lambda \frac{\frac{k_{i 1}}{w_{c}^{2}}}{1+\left(\frac{k_{i 1}}{w_{c}}\right)^{2}}-A=0
$$

$k_{i 4}$ represents the solution of the second-class equation (14)

$$
A k_{i 1}^{2}-\lambda k_{i 1}+A w_{c}^{2}=0
$$

while

$$
A=\frac{\alpha T w_{c}^{\alpha-1} \sin \frac{\alpha \pi}{2}}{\left(T w_{c}^{\alpha} \sin \frac{\alpha \pi}{2}\right)^{2}+\left(1+T w_{c}^{\alpha} \sin \frac{\alpha \pi}{2}\right)}
$$

The resolution of this equation allows writing $k_{d 3}$ according to $\lambda$

Finally we can deduct from the last condition (8) the value of $k_{p 1}$.

$$
\begin{gathered}
\left|G_{1}\left(j w_{c}\right)\right|=\left|C_{1}\left(j w_{c}\right)\right|\left|P\left(j w_{c}\right)\right|=\left|\frac{k_{p 1}\left(1+\left(\frac{-k_{i 1}}{w_{c}}\right)^{2}\right)^{\frac{\lambda}{2}}}{N}\right|=1 \\
\Rightarrow k_{p 1}=\left|\frac{N}{k_{p 1}\left(1+\left(\frac{-k_{i 1}}{w_{c}}\right)^{2}\right)^{\frac{\lambda}{2}}}\right|
\end{gathered}
$$

Avec

$$
N=\sqrt{\left(T w_{c}^{\alpha+1} \cos \frac{\alpha \pi}{2}+w_{c}\right)^{2}+\left(T w_{c}^{\alpha+1} \sin \frac{\alpha \pi}{2}\right)^{2}}
$$

A graphics method to find the two first parameters of the regulator consists in drawing both functions of $k_{i 1}$ according to $\lambda$, the intersection of these two curves gives the values of $k_{i 1}$ and which verify both equations.

The figure below represents both curves describing the variation of $k_{i 1}$ according to $\lambda$.

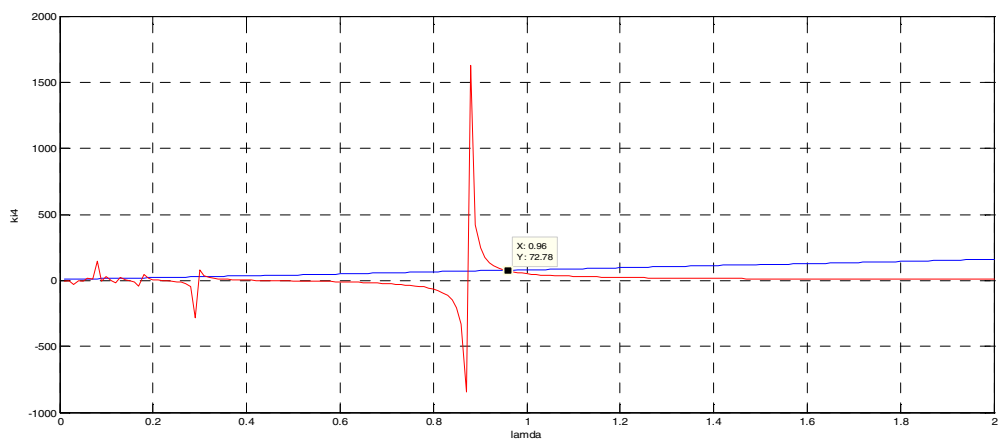

Figure 1. Variation of $k_{i 1}$ according to $\lambda$ 
The intersection gives $k_{i 1}=72.78, \lambda=0.96$ from the equation (16), we deduct $k_{p 1}=7.8198$, and the synthesized regulator has the following shape.

$$
C_{1}(s)=13.9969\left(1+\frac{72.78}{s}\right)^{0.96}
$$

The diagram of bode of the open loop is represented by the figure below.
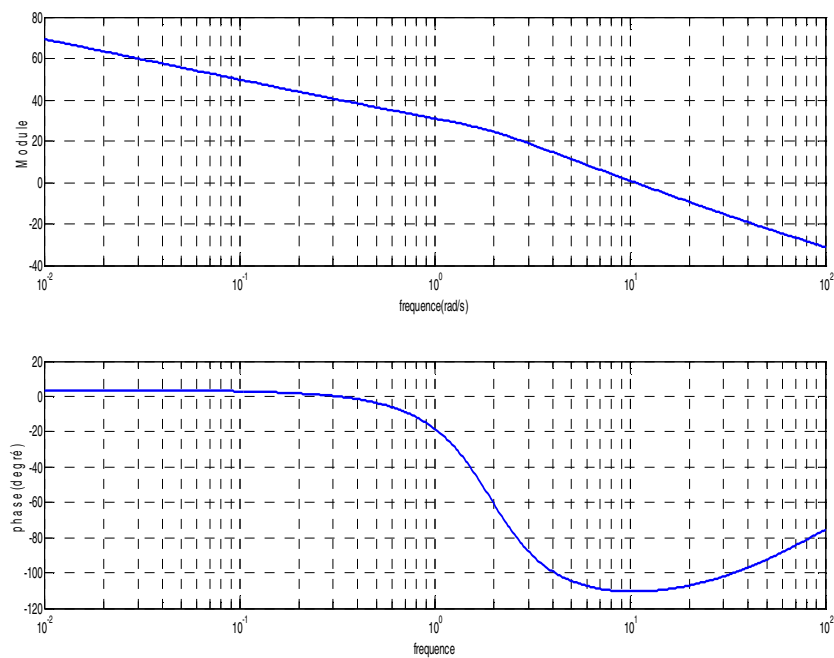

Figure 2. Bode Diagramm fo the FO-[P I]

We observe that the performances wished are respected; indeed the margin of phase is equal to $70^{\circ}$ at the corner frequency.

\section{B. Integer Order [PI] controller design}

The classical controller PI parameters are obtaining using the classical PI controller design techniques which are obtained in [12] for integer order interval control systems, are extended for the FOS.

This method is a little modified compared with the previous one but both arise from the same idea which is based on the frequency analyzes of the open loop before and after the integration of the regulator. The regulator PI has the following shape.

$$
C_{2}(s)=k_{p 2}+\frac{k_{i 2}}{s}
$$

The gain $k_{p 2}$ of this controller can be calculated from the expression of the amplitude of the system with the new corner frequency which gives the margin of the wished phase; so that the gap of phase of the compensator does not affect the phase of the system the report $\frac{k_{i 2}}{k_{p 2}}$ will be chosen to be equal to a ten decade below $w_{c}^{\prime}$.
From the figure (1) illustrating the diagram bode of the transfer function of the system (1), the corner frequency $w_{c}$ can be varied towards the new frequency $w_{c}^{\prime}$ which corresponds to the required phase.

The value of $k_{p 2}$ and $k_{i 2}$ can be calculated from the equation (19)

$$
\left|G_{2}\left(j w_{c}^{\prime}\right)\right|=-20 \log \left(k_{p 2}\right)=a d B, k_{i 2}=\frac{w_{c}^{\prime}}{10} k_{p 2}
$$

According to the bode diagram of the system (1), the margin of phase wished $\left(70^{\circ}\right)$ is finished with the frequency $w_{c}^{\prime}=0.5909$, the gain at this frequency is equal to $2.738 \mathrm{~dB}$, the equation (19) allows to calculate the parameters of the regulator

$$
k_{p 2}=10^{\left(\frac{-2.738}{20}\right)}=0.7296 \quad k_{i 2}=\frac{\mathrm{w}_{\mathrm{c}}^{\prime} k_{p 2}}{10}=0.0431
$$

The expression of the regulator $\mathrm{C}_{2}(\mathrm{~s})$ has then the shape (20):

$$
\mathrm{C}_{2}(\mathrm{~s})=\frac{0.7296 s+0.0431}{s}
$$

The bode diagram of open loop after the integration of the previous regulator is presented by the figure (3).
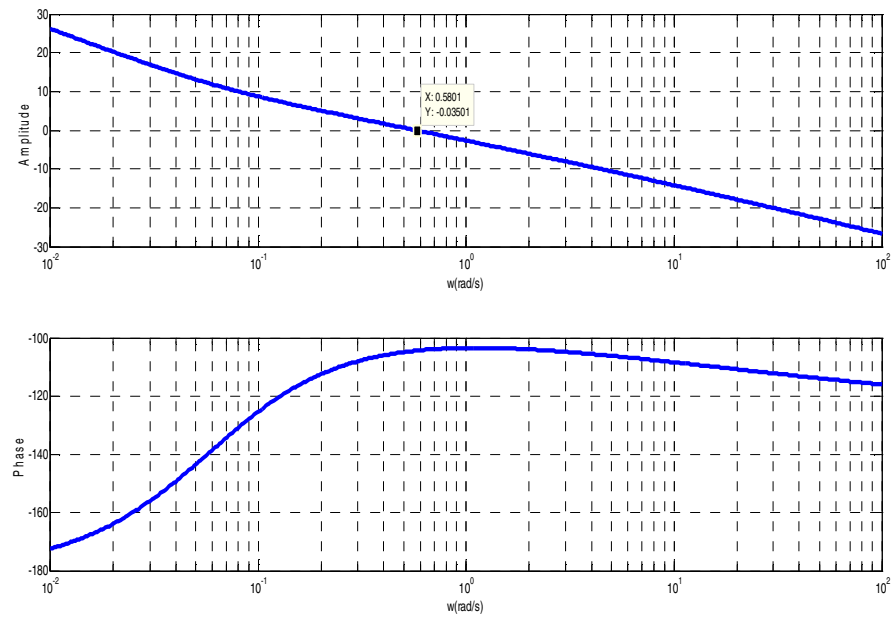

Figure 3. Bode diagram of the system $+\mathrm{IO}[\mathrm{PI}]$

We notice that the wished performances are established, indeed, the amplitude nullifies with the new corner frequency chosen and the margin of the phase wished is finished at this frequency. 


\section{State SPace RePRESENTATION OF THE FOS}

To study the stability performances of the system we will analyze the temporal response.

In this paragraph, we extended the base form of commandability applied to integer order systems to fractional systems using a fractional integrator.

Let us consider as fractional transfer function the following shape:

$$
H(s)=\frac{b_{m} s^{m}+b_{m-1} s^{\lambda}+\ldots+b_{1} s^{\mu}+b_{0}}{s^{\alpha}+a_{n-1} s^{\beta}+\ldots+a_{1} s^{\delta}+a_{0}}
$$

By multiplying at the top and below by $s^{-\alpha}$ the function (21) can be rewritten as follows:

$$
H(s)=\frac{b_{m}\left(\frac{1}{s}\right)^{\alpha-m}+\ldots+b_{1}\left(\frac{1}{s}\right)^{\alpha-\mu}+b_{0}\left(\frac{1}{s}\right)^{\alpha}}{1+a_{n-1}\left(\frac{1}{s}\right)^{\alpha-\beta}+\ldots+a_{1}\left(\frac{1}{s}\right)^{\alpha-\delta}+a_{0}\left(\frac{1}{s}\right)^{\alpha}}
$$

We put then

$$
\begin{gathered}
H(s)=\frac{Y(s)}{U(s)}=\frac{Y(s)}{W(s)} \frac{W(s)}{U(s)} \\
\frac{Y(s)}{W(s)}=N(s) \text { et } \frac{W(s)}{U(s)}=\frac{1}{D(s)}
\end{gathered}
$$

with $\quad N(s)=b_{m}\left(\frac{1}{s}\right)^{\alpha-m}+\ldots+b_{1}\left(\frac{1}{s}\right)^{\alpha-\mu}+b_{0}\left(\frac{1}{s}\right)^{\alpha}$

$$
D(s)=1+a_{n-1}\left(\frac{1}{s}\right)^{\alpha-\beta}+\ldots+a_{1}\left(\frac{1}{s}\right)^{\alpha-\delta}+a_{0}\left(\frac{1}{s}\right)^{\alpha}
$$

The system output will have the shape (24)

$Y(s)=W(s)\left[b_{m}\left(\frac{1}{s}\right)^{\alpha-m}+\ldots+b_{1}\left(\frac{1}{s}\right)^{\alpha-\mu}+b_{0}\left(\frac{1}{s}\right)^{\alpha}\right]$

By means of this expression we can deduct the base form of commandability which allows feigning on Matlab any system having an explicit transfer function using a simulink block of a fractional integrator predefines. Using the previous equations we can extract the following general representation of a FOS.

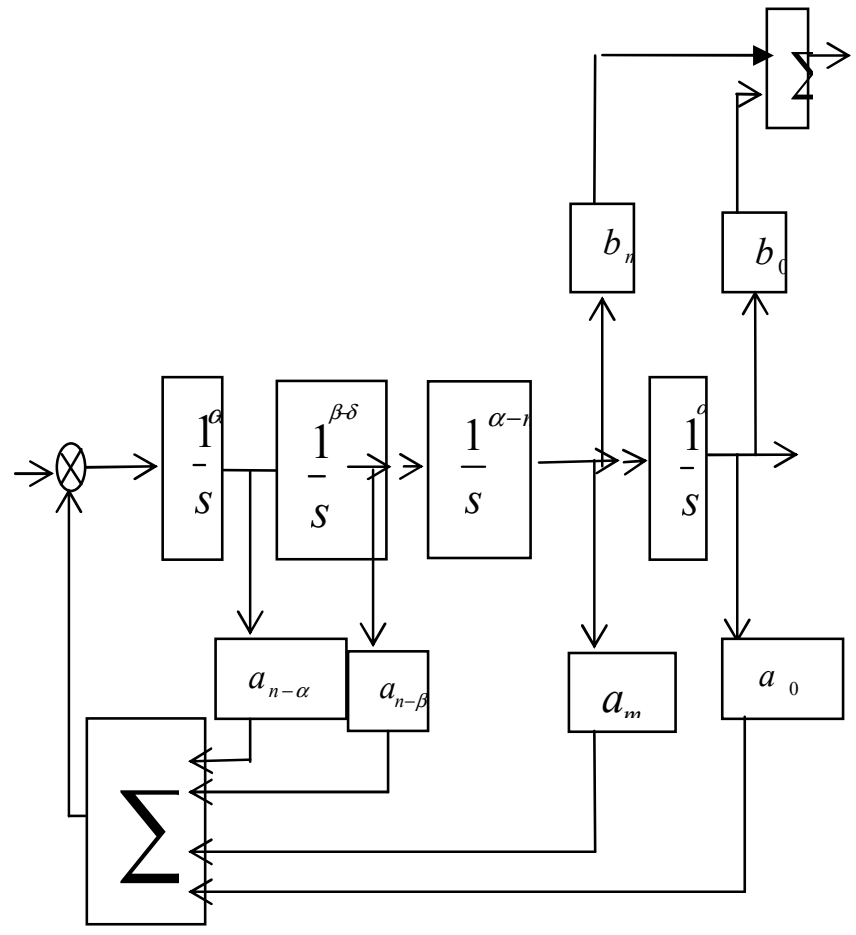

Figure 6. General representation of a FOS

V. NUMERICAL Simulations

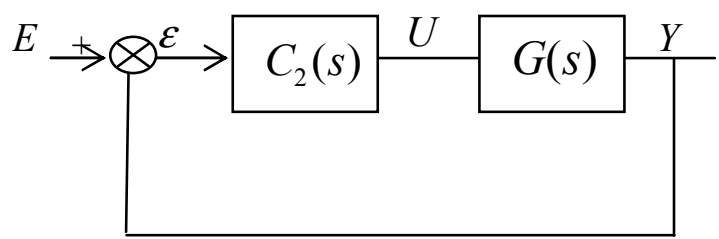

Figure 4. closed loop system shématic

We feign the system with negative feedback by applying him a unit step as input figure (4). The step response of the system with the first regulator $\mathrm{FO}[\mathrm{PI}]$ and the second regulator $\mathrm{IO}[\mathrm{PI}]$ are respectively illustrated in figures (5) and (6). 


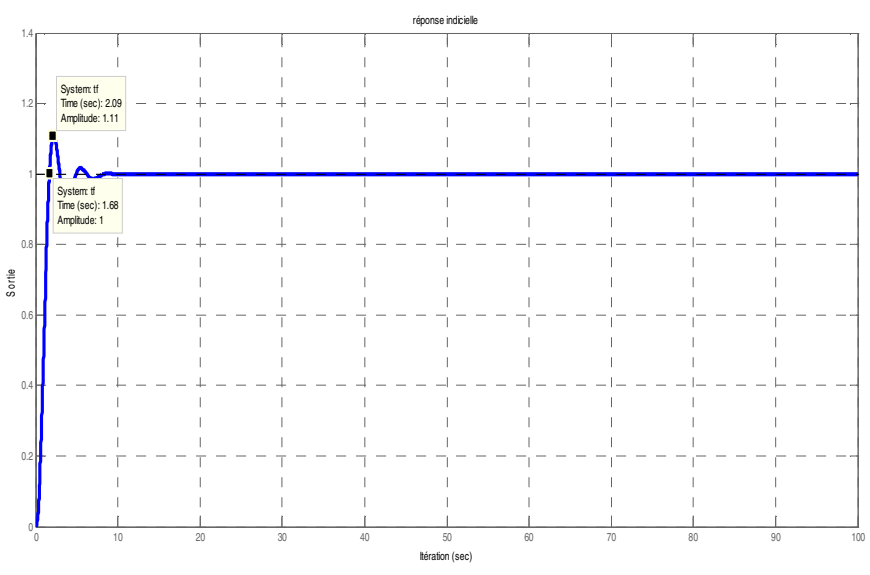

Figure 5. Simulation step response of the système+ FO-[PI]

The regulator FO-[ PI] insures the stability of the system with an overtaking of the order of 0.11 , the necessary time to reach the gain input value for the first time is $t=1.68 \mathrm{~s}$ and a zero as static error .

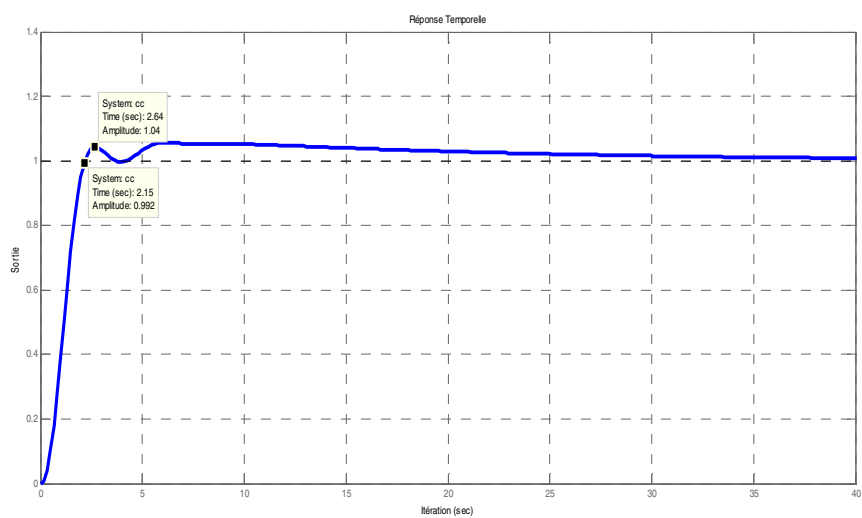

Figure 6. Simulation. step responses of the système+ $\mathrm{IO}[\mathrm{PI}]$

The integer regulator PI assures the stability of the system with an overtaking $d=0.06$, a zero static error and a time $t=2.19 \mathrm{~s}$ to reach for the first time the gain input value.

\section{COMPARISON RESULTS}

To judge the robustness of the system for discussing regulator, four criteria of optimizations will be considered. The following table illustrates the results found for each regulator.

TABLE I. COMPARAISON RESULTS

\begin{tabular}{|l|l|l|l|l|}
\hline & ISE & IAE & ITAE & Overtaking \\
\hline $\begin{array}{l}\text { Compensator } \\
\text { IO-PI }\end{array}$ & 0.0187 & 0.1368 & 6.6259 & 0.06 \\
\hline FO-PI & 0.0118 & 0.1085 & 0.0308 & 0.1 \\
\hline
\end{tabular}

According to the board, it is clear that the regulator FO$[\mathrm{PI}]$ is the best synthesized regulator from the two, seen that it presents the minimum of three criteria illustrated in the board. According to the overtaking, the $\mathrm{IO}[\mathrm{PI}]$ present the lower value compared with the FO [PI].

\section{CONCLUSION}

In this paper, we defined a fractional system, we analyzed its frequency performances without regulator, then we synthesized the necessary regulators; two methods are presented for controller design in the presence of a fractional case; the first method presents a robust parametric fractional controller design to satisfy robust stability and the required performance specifications of the fractional order plant, the second presents a robust parametric classical design PI.

Then, we applied the command by these two regulators to the same system predefines and we feigned the step response of the closed loop. Best results were obtained with fractional regulators $\mathrm{FO}[\mathrm{PI}]$.

\section{REFERENCES}

[1] :I.Podbuly,"Fractional Differential Equations",Academic Pres,San Diego,1999

[2]:T.F.Nonnenmacher and W.G Glockle,"A Fractional model for mechanical stress relaxation," Philosophical Magazine

Lett,vol.64,no.2,pp.89-93,1991

[3]:S.Westerlund,"Capacitor theory," IEEE Trans. Dielec.Elec.Ins,vol. 1, no.5,pp.826-839,1994

[4]: Mandelbrot B.B, Some noises with $1 / \mathrm{f}$ spectrum, a bridge between direct current and white noise, IEEE Trans. Infor.Theory, vol.IT-13, $\mathrm{N}^{\circ} 2$, April 1967

[5]: Mandelbrot B,B.,The fractional Geometry of Nature,W.H. Freeman\& Co, 1983

[6]:Conception A.Monje YangQuan Chen Blas M. Vinagre Dingyu Xue. Vicente Feliu "Fractional-order Systems and Controls" Springer,Scotlande,UK,2010

[7] A.Oustaloup "Systèmes asservis linéaires d'ordre fractionnaires" Masson.Paris, 1983

[8] :J.Sabatier, S.Poullain, P. Latteux, J.L. Thomas, and A. Oustaloup “ Robust speed control of a low damped electromechanical system based on CRONE control: application to a four mass experimental test bench," Nonlinear Dynamics, vol. 38, pp.383-400,2004.

[9] :C.A.Monje, B.M.Vinagre, and V. Feliu, et. Al, "Tuning and autotuning of fractional order controllers for industry applications," Control Engineering Practice, vol. 16, pp.798-812, 2008.

[10] :Ying Luoa,b, Yang Quan Chenb "Fractional order [Prportional derivative] controller for a class of fractional order system, "USA, 2009.

[11] :Celaleddin Yergolo,M. Mine Ozyetkin, and Nusret Tun

"Frequency Response Computation of Fractional Order Interval Transfer Functions ",2010

[ 12] :Bhattacharyya SP, Chapellat H, Keel LH. Robust control: the parametric approach.Prentice Hall;1995

[12]: Valerio D., da Costa J. S. Tuning of fractional PID controllers with ziegler-Nichols-type rules. Signal Processing. 